\title{
Increased ocular levels of IGF-1 in transgenic mice lead to diabetes-like eye disease
}

\author{
Jesús Ruberte,1,2 Eduard Ayuso,1,3 Marc Navarro,1,2 Ana Carretero,1,2 Víctor Nacher,1,2 \\ Virginia Haurigot,, 1,3 Mónica George, 1,3 Cristina Llombart,, ${ }^{1,2}$ Alba Casellas,, ${ }^{1,3}$ Cristina Costa, ${ }^{3}$ \\ Assumpció Bosch,1,3 and Fatima Bosch ${ }^{1,3}$
}

${ }^{1}$ Center of Animal Biotechnology and Gene Therapy, ${ }^{2}$ Department of Animal Health and Anatomy, School of Veterinary Medicine, and

${ }^{3}$ Department of Biochemistry and Molecular Biology, Universitat Autònoma de Barcelona, Bellaterra, Spain.

\begin{abstract}
IGF-1 has been associated with the pathogenesis of diabetic retinopathy, although its role is not fully understood. Here we show that normoglycemic/normoinsulinemic transgenic mice overexpressing IGF-1 in the retina developed most alterations seen in human diabetic eye disease. A paracrine effect of IGF-1 in the retina initiated vascular alterations that progressed from nonproliferative to proliferative retinopathy and retinal detachment. Eyes from 2-month-old transgenic mice showed loss of pericytes and thickening of basement membrane of retinal capillaries. In mice 6 months and older, venule dilatation, intraretinal microvascular abnormalities, and neovascularization of the retina and vitreous cavity were observed. Neovascularization was consistent with increased IGF-1 induction of VEGF expression in retinal glial cells. In addition, IGF-1 accumulated in aqueous humor, which may have caused rubeosis iridis and subsequently adhesions between the cornea and iris that hampered aqueous humor drainage and led to neovascular glaucoma. Furthermore, all transgenic mice developed cataracts. These findings suggest a role of IGF-1 in the development of ocular complications in long-term diabetes. Thus, these transgenic mice may be used to study the mechanisms that lead to diabetes eye disease and constitute an appropriate model in which to assay new therapies.
\end{abstract}

\section{Introduction}

Diabetic eye disease develops as a complication of long-term diabetes and is the most common cause of blindness. It includes retinopathy and, less frequently, rubeosis iridis, neovascular glaucoma, and cataracts (1). Diabetic retinopathy begins with a nonproliferative phase involving increased vascular permeability, thickening of the basement membrane, and loss of pericytes in the retinal capillaries. It progresses to the proliferative phase, in which neovascularization causes visual impairment. The severity increases as diabetes progresses, and nearly all diabetic patients develop retinopathy within 20 years of diagnosis, half of which have proliferative retinopathy (2). The current treatments for diabetic retinopathy, laser photocoagulation of the retina and vitrectomy, are invasive and provide only temporary protection (3). Development of more effective therapies requires both understanding of the mechanisms that lead to the disease and the development of animal models in which to assay new treatments. The existing animal models of diabetes do not develop proliferative retinopathy, and a widely used model, which mimics the neovascularization noted in human diabetic patients, is induced by relative hypoxia in developing retinas (4).

Several growth factors have been implicated in the pathogenesis of diabetic eye disease. An increase of IGF-1 has been found in the vitreous of patients with diabetic retinopathy (5-7). IGF-1 receptors are widely distributed in the eye (8), and treatment of ischemia-induced mice with IGF-1 receptor antagonist prevents

Nonstandard abbreviations used: glial fibrillar acidic protein (GFAP); intraocular pressure (IOP); intraretinal microvascular abnormalities (IRMAs); rat insulin promoter-I (RIP-I); streptozotocin (STZ); von Willebrand factor (vWF).

Conflict of interest: The authors have declared that no conflict of interest exists.

Citation for this article: J. Clin. Invest. 113:1149-1157 (2004).

doi:10.1172/JCI200419478. retinal neovascularization (9), which indicates a key role of IGF-1mediated signaling. Furthermore, following hypoxia, vascular endothelial cell-specific IGF-1 receptor KO mice (VENIFARKO) show reduced retinal neovascularization (10). Similarly, in humans, mutations in growth-hormone receptor, IGF-1, or IGF-1 receptor genes reduce retinal vascularization (11). The source of increased ocular IGF-1 in retinopathy is controversial, and the relative contribution of either endogenous IGF-1 (12) or serum IGF-1 is unknown. Increased IGF-1 serum levels during pregnancy in type 1 diabetes correlate with progression of diabetic retinopathy (13). Similarly, the paradoxical initial increase in retinal neovascularization observed shortly after intensive insulin therapy in patients with poorly controlled diabetes is consistent with the rise in serum IGF-1 levels (14-20). Moreover, hypoxia-stimulated retinal neovascularization is inhibited in mice expressing a growth-hormone antagonist gene or in normal mice given an inhibitor of growth-hormone secretion, both leading to low circulating IGF-1 (21). Exogenous IGF-1 administration reverses this phenotype (21).

VEGF promotes retinal neovascularization and is considered a key factor in the progression of retinopathy (22-25). Moreover, the expression of VEGF in the retina of transgenic mice leads to retinal neovascularization $(26,27)$. The lack of IGF-1 in $\mathrm{KO}$ mice, however, prevents normal retinal vascular growth, despite the presence of VEGF (28). Moreover, IGF-1 induces VEGF expression in several cell types and may also enhance VEGF signaling $(9,29,30)$. Thus, both factors may be complementary for retinal neovascularization in diabetic retinopathy. Nevertheless, there is no direct evidence for the role of IGF-1 in the initiation of diabetic retinopathy. Here we show that transgenic mice overexpressing IGF-1 in the retina progressively develop retinal neovascularization associated with rubeosis 
A

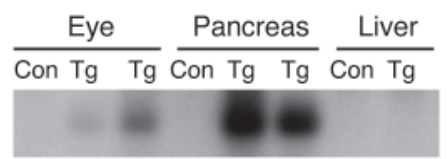

B

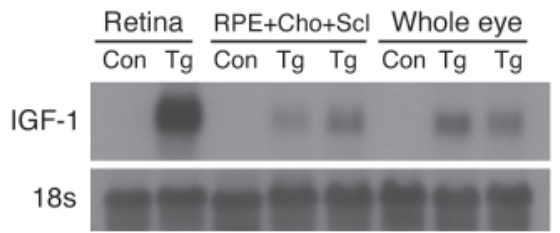

C
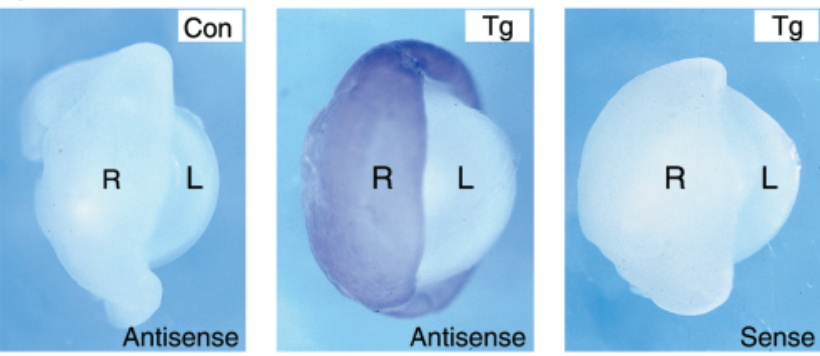

D

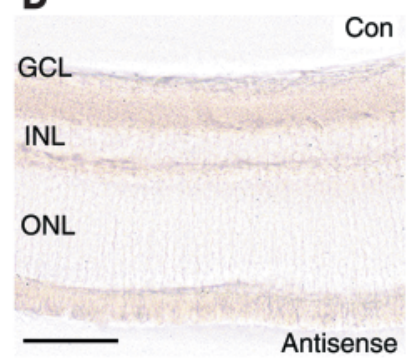

Con

$\operatorname{Tg}$

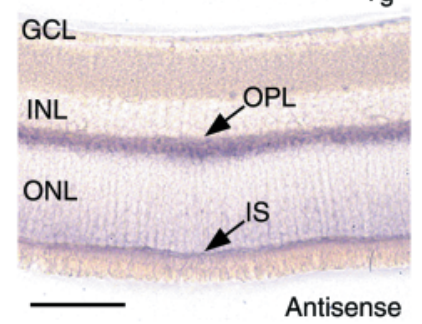

iridis, neovascular glaucoma, and cataracts, indicating that an increase in ocular IGF-1 may lead to diabetes-like eye disease.

\section{Methods}

Animals. Male transgenic mice overexpressing IGF-1 under the control of the rat insulin promoter-I (RIP-I) in both the C57BL/6-SJL and CD1 mouse genetic background (31), which showed similar phenotype, were used. The expression of IGF-1 in $\beta$ cells of transgenic mice counteracts cytotoxicity and insulitis after treatment with multiple low doses of streptozotocin (STZ), increases $\beta$ cell mass through neogenesis and $\beta$ cell replication, and leads to recovery from type 1 diabetes (31). Mice were fed ad libitum with a standard diet (Panlab SL, Barcelona, Spain). Animal care and experimental procedures were approved by the Ethics Committee in Animal and Human Experimentation of the Autonomous University of Barcelona.

Analysis of IGF-1,VEGF, and glial fibrillar acidic protein expression. Ten micrograms of total RNA from whole eye or from different ocular structures were analyzed by Northern blot. RT-PCR analysis of RNA from the whole eye was carried out by standard methods. The RIP-I/IGF-1 chimeric gene contained partial rabbit $\beta$-globin sequences that were used to design specific primers (31). The forward primer was in exon 2 of $\beta$ globin (5'-GGATCCTGAGAACTTCAG-3') and the reverse primer was inside the IGF-1 cDNA (5'-TTCCTGCACTTCCTCTAC-3'). PCR-amplified products, transferred to membranes, and Northern blots were

\section{Figure 1}

IGF-1 is overexpressed in eyes of transgenic mice. (A) RT-PCR confirmed that IGF-1 in the eye was a result of transgene expression. Pancreas mRNA was used as a positive control and liver mRNA as a negative control. The amplified band hybridized with an IGF-1 CDNA probe confirms the specificity of the RT-PCR. (B) Northern blot analysis was performed using samples from retina, retinal pigmented epithelium (RPE), choroid (Cho), sclera (Scl), and from the whole eye. Endogenous IGF-1 was detected only after long exposure (data not shown). (C) IGF-1 expression in transgenic retina by in situ hybridization. Sense probe did not give any signal. (D) In the transgenic retina, purple staining indicated expression of IGF-1 in the outer plexiform layer and in the inner segment of photoreceptors. No IGF-1 signal was observed in the retina of control mice. Con, control; GCL, ganglion cell layer; INL, inner nuclear layer; IS, inner segment of photoreceptors; L, lens; ONL, outer nuclear layer; OPL, outer plexiform layer; R, retina; Tg, transgenic. Scale bars: $72 \mu \mathrm{m}$.

hybridized with a mouse IGF-1 or VEGF 164 (kindly provided by P.A. D'Amore, Harvard Medical School, Boston, Massachusetts, USA) cDNA probes. For in situ hybridization, mouse IGF-1 cDNA was cloned into PSPT18 vector, and sense and antisense digoxigenin-labeled probes were generated from SP6 and T7 promoters following the manufacturer's instructions (Roche Molecular Biochemicals, Mannheim, Germany). In situ hybridization was carried out on whole-mount dissected eyes (32). The IGF-1 concentration in the aqueous humor was measured by a rat IGF-1 enzyme immunoassay (Diagnostic Systems Laboratories Inc., Webster, Texas, USA). To determine VEGF and glial fibrillar acidic protein (GFAP) expression, whole eyes were homogenized in lysis buffer. Western blot analysis was performed on $300 \mu \mathrm{g}$ of protein using a rabbit anti-cow GFAP (DAKO Corp., Carpinteria, California, USA), a goat anti-mouse VEGF, or a rabbit antiactin Ab (Sigma-Aldrich, St. Louis, Missouri, USA).

Immunohistochemistry. Whole-mount formalin-fixed retinas or tissue sections $(2 \mu \mathrm{m})$ were incubated with rabbit anti-human VEGF Ab (Santa Cruz Biotechnology Inc., Santa Cruz, California, USA) at 1:50 dilution, goat anti-mouse VEGF Ab (Sigma-Aldrich) at 1:100 dilution, rabbit anti-cow GFAP (DAKO Corp.) at 1:500 dilution, rabbit anti-mouse collagen IV Ab (Chemicon International, Temecula, California, USA) at 1:100 dilution, rabbit antihuman von Willebrand factor (vWF) (DAKO Corp.) at 1:500 dilution, and biotinylated Griffonia simplicifolia lectin I $(20 \mu \mathrm{g} / \mathrm{ml}$; Vector Laboratories, Burlingame, California, USA). As secondary Ab's, biotinylated anti-rabbit IgG (Vector Laboratories) and

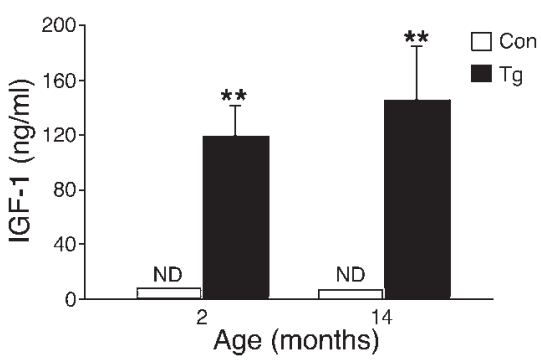

\section{Figure 2}

IGF-1 levels in aqueous humor. IGF-1 was detected by ELISA in the aqueous humor of 2- and 14 month-old transgenic mice, but not in controls (ND, not detected). Results are mean \pm SEM of eight mice in each group. ${ }^{* *} P<0.01$. 
A

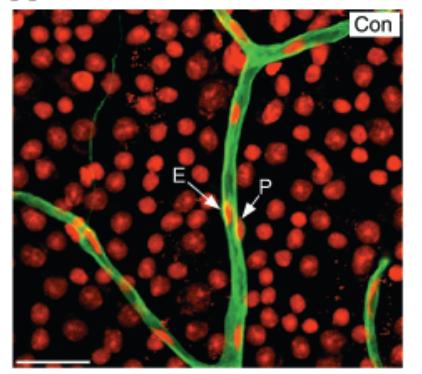

B
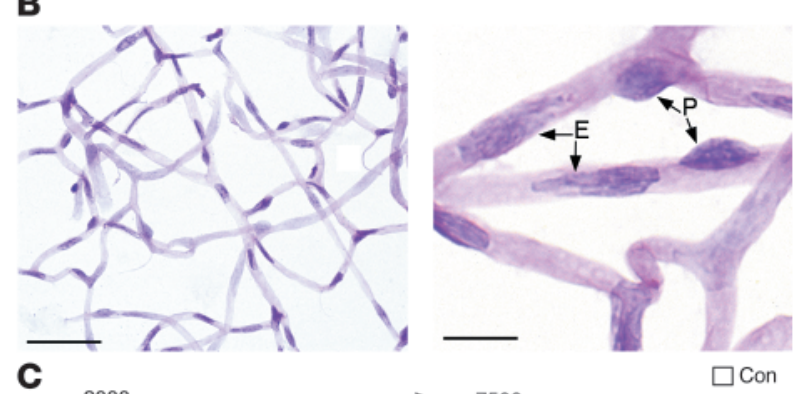

C
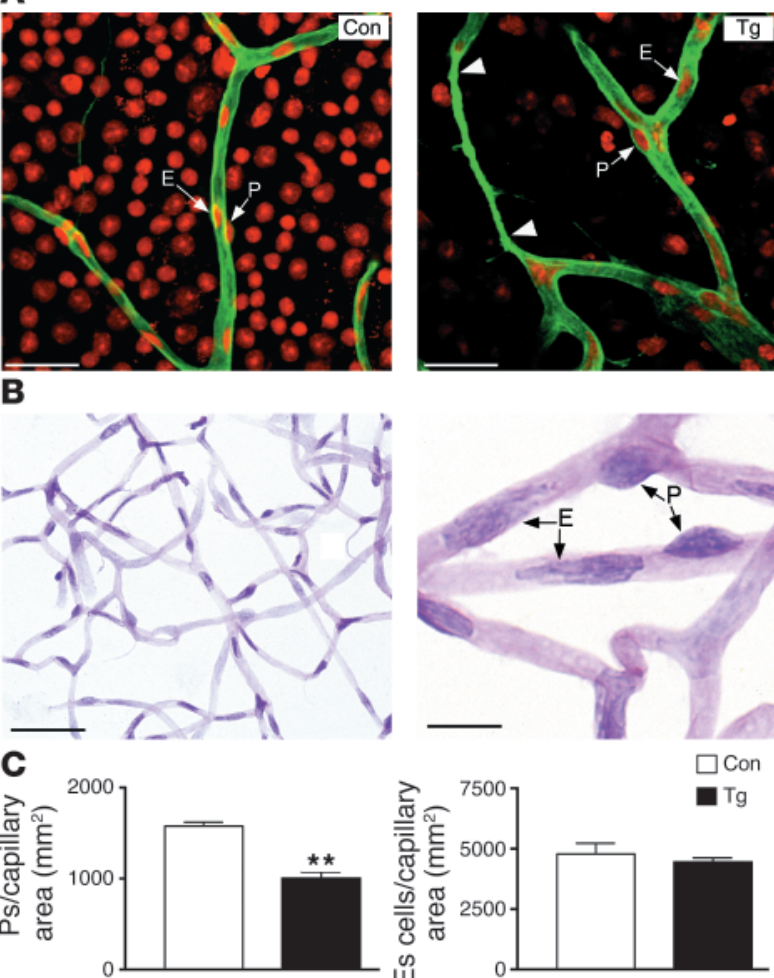

$\mathrm{Tg}$

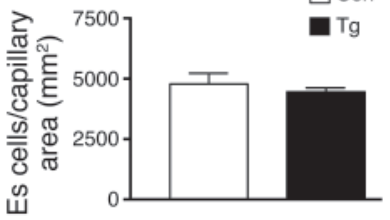

biotinylated anti-goat IgG (Santa Cruz Biotechnology Inc.) were used. Streptavidin Alexa Fluor 488 conjugate and streptavidin rhodamine conjugate as fluorochromes (Molecular Probes Europe BV, Leiden, The Netherlands) and propidium iodide for nuclear counterstaining (Sigma-Aldrich) were used for laser-scanning confocal analysis (TCs SP2; Leica Microsystems GmbH, Heidelberg, Germany). Alternatively, ABC complex (Vector Laboratories) and $3^{\prime} 3^{\prime}$-diaminobenzidine (Sigma-Aldrich) were used. Sections were counterstained in Mayer's hematoxylin.

Retinal digest preparations. Retinas were obtained after enucleation of eyes and were immediately fixed in $10 \%$ formalin in phosphate buffer. Retinal vascular preparations were performed using a pepsin-trypsin digestion technique (33). Briefly, a pepsin digestion ( $5 \%$ pepsin in $2 \%$ hydrochloric acid for 15 minutes at $37^{\circ} \mathrm{C}$ ) combined with a trypsin digestion ( $3 \%$ in PBS for 3 hours at $37^{\circ} \mathrm{C}$ ) was used to isolate the retinal vasculature. Afterward, the retinal preparations were stained with periodic acid-Schiff reagent and hematoxylin.

\section{Figure 4}

Thickening of capillary basement membrane was observed in transgenic retinas. (A) Confocal analysis of retinal capillaries in paraffin sections of retinas immunohistochemically marked with anti-collagen IV (green). Nuclei were counterstained with propidium iodide (red). (B) Transmission electron microscopic analysis of retinal capillaries (upper panels). Thickening of basement membrane (arrows) of transgenic retinas is shown (lower panels, magnification of insets in corresponding upper panels). (C) The basal membrane thickness was measured as indicated in Methods. Results are mean \pm SEM of five mice in each group. ${ }^{\star \star} P<0.01$. Er, erythrocyte. Scale bars: $5.6 \mu \mathrm{m}(\mathbf{A}), 2.7 \mu \mathrm{m}(\mathbf{B}$, upper panel), and $487 \mathrm{~nm}$ (B, lower panel).

\section{Figure 3}

Pericyte loss in retinal capillaries from transgenic mice overexpressing IGF-1. (A) Confocal analysis of retinal capillaries in flat-mounted retinas immunohistochemically marked with anti-collagen IV (green). Nuclei were counterstained with propidium iodide (red). Acellular capillaries, lacking pericytes and endothelial cells, were observed (arrowheads) in transgenic retina. The images are single confocal sections. (B) Retinal digest preparations were obtained as indicated in Methods. A representative area is shown (left panel). Endothelial cell nuclei are placed within the vessel wall, while pericyte nuclei are placed more laterally on the vessel wall (right panel). (C) The number of pericytes (left) and endothelial cells (right) per square millimeter of capillary area were determined as indicated in Methods. Results are mean \pm SEM of five mice in each group. ${ }^{* \star} P<0.01$. E, endothelial cell; P, pericyte. Scale bars: $35 \mu \mathrm{m}$ (A), $43 \mu \mathrm{m}$ (B, left panel), and $11 \mu \mathrm{m}$ (B, right panel).

Transmission electron microscopic analysis. Retinas were obtained and a peripheral area of $1 \mathrm{~mm}^{2}$ was cut and fixed in $2.5 \%$ glutaraldehyde and $2 \%$ paraformaldehyde for 2 hours at $4{ }^{\circ} \mathrm{C}$. After washing in cold cacodylate buffer, the specimens were post-fixed in $1 \%$ osmium tetroxide, stained in aqueous uranyl acetate, and then dehydrated through a graded ethanol series and embedded in epoxy resin. Ultrathin sections (600-800 $\mathrm{A}$ ) from the resin blocks were stained using lead citrate and examined in a transmission electron microscopy (Hitachi H-7000; Hitachi Ltd., Tokyo, Japan).

Retinal fluorescein angiograms. The thoracic aorta was cannulated and injected with $1 \mathrm{ml}$ of FITC-dextran (Sigma-Aldrich). Eyes were fixed in $10 \%$ formalin in phosphate buffer, and retinas were isolated, flattened, and observed in a epifluorescence microscope (Eclipse E800; Nikon Corp., Tokyo, Japan).

Scanning electron microscopic analysis. Eyes were dissected and fixed in $2 \%$ paraformaldehyde and $0.5 \%$ glutaraldehyde. After dehydration,

A
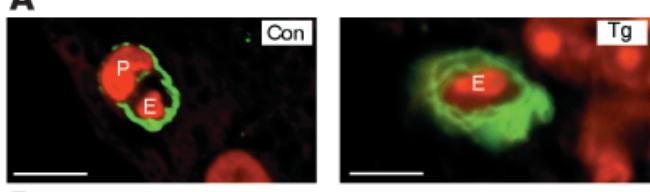

B
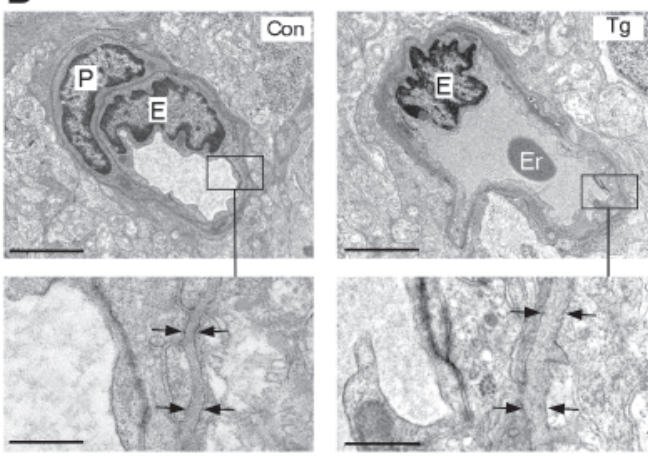

C

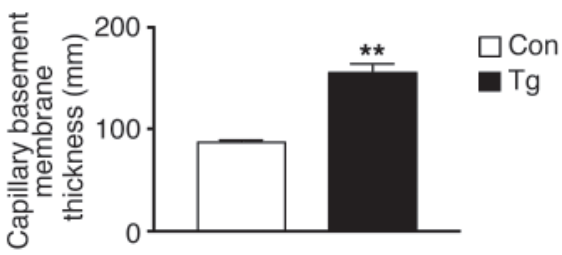


A

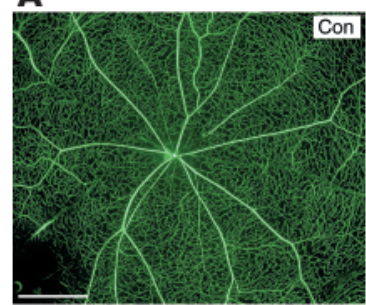

B

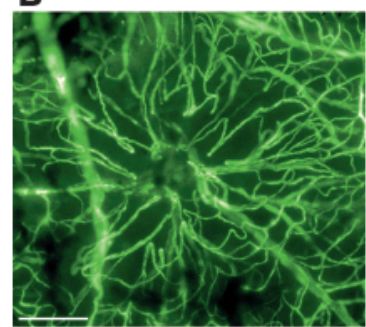

D

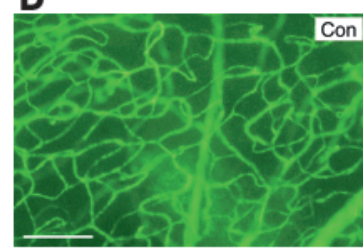

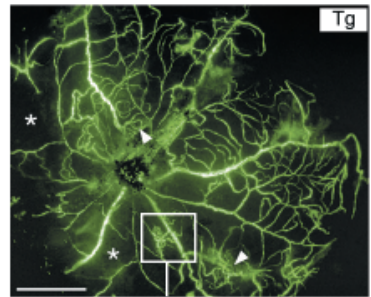

c
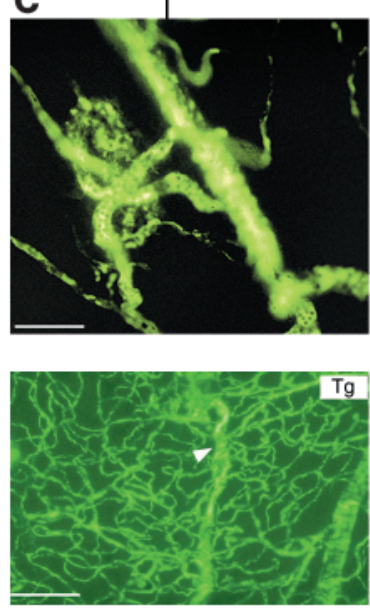

samples were critical-point dried, mounted on stubs, coated with gold-palladium, and observed in a scanning electron microscope (Hitachi S570; Hitachi Ltd.) at an acceleration voltage of 15-20 kV. Vascular corrosion casts were performed by injection of Mercox resin (Ladd Research, Williston, Vermont, USA) in the thoracic aorta. After corrosion of eyes in $5 \% \mathrm{KOH}$, vascular casts were dried, mounted on stubs, gold-palladium coated, and observed at an acceleration voltage of 5-10 kV.

Intraocular pressure measurements in vivo. Mice were anesthetized with a mixture of $10 \mathrm{mg} / \mathrm{kg}$ xylazine and $100 \mathrm{mg} / \mathrm{kg}$ ketamine, and intraocular pressure (IOP) was measured in the right eye by an induction-impact tonometer (34) (ICare, Tiolat Ltd., Helsinki, Finland). Six tonometer measurements were performed in each eye.

Morphometric analysis. To determine pericyte and endothelial cell loss in transgenic retinas, the total number of pericytes and endothelial cells was counted in ten randomly selected microscopic fields from each retinal-digest preparation. The cell numbers obtained were normalized to the relative capillary density (numbers of cells per square millimeter of capillary area). Quantification of the retinal capillary basement membrane thickness was always performed in the periphery of the retina to avoid the effect of retinal site on the basement membrane thickness (35).

\section{Figure 6}

Neovessels in the vitreous cavity of transgenic eyes. (A) Blood vessels inside the vitreous (left panel) were immunodetected (right panel, magnification of inset in left panel) with anti-vWF (green). Nuclei were counterstained with propidium iodide. (B) Scanning electron microscopy analysis of Mercox vascular casts from an intravitreous neovessel (left panel) and intraretinal normal vessel (right panel). The intravitreous neovessels showed imprints of endothelial nuclei (arrowheads), which appeared to be more prominent and numerous than intraretinal vessels. Scale bars: $36 \mu \mathrm{m}(\mathbf{A})$ and $11 \mu \mathrm{m}$ (B).

\section{Figure 5}

IRMAs and neovessels in transgenic retina. (A) Flat-mounted, FITCdextran-perfused retinas from transgenic eyes showed extensive areas of non-perfusion (asterisks) and neovessels (arrowheads and inset). (B) Capillary IRMA inside the retina is shown. (C) Neovessel originating from a venule (magnification of inset in $\mathbf{A}$, right panel). This vessel had more endothelial cell nuclei after staining with propidium iodide and analysis with laser confocal microscopy (data not show). (D) Intraretinal dense area of neocapillaries in transgenic retina originating from a central feeding vessel (arrowhead). Scale bars: $630 \mu \mathrm{m}$ (A), $188 \mu \mathrm{m}$ (B), $87 \mu \mathrm{m}$ (C), and $105 \mu \mathrm{m}$ (D).

Five capillaries per retina, identified by morphology and size, were photographed at $\times 20,000$. Twelve measurements of basement membrane were taken in each capillary where the cytoplasmic cell membranes, bordering the basement membrane, were sharp black lines. To determine venule dilatation, the maximal venule diameter was measured in FITC-dextran-injected retinas. Morphometric analyses were performed using an image analyzer (analy-SIS 3.0; Soft Imaging System Corp., Lakewood, Colorado, USA).

Statistical analysis. All values were expressed as mean \pm SEM. Twotailed $P$ values were calculated by unpaired Student $t$ tests. Differences were considered statistically significant at $P$ values less than 0.05 .

\section{Results}

IGF-1 is overexpressed in eyes of transgenic mice. Transgenic mice overexpressing IGF-1 under the control of the RIP-I show increased IGF-1 in $\beta$ cells (31). These mice are normoglycemic and normoinsulinemic and have body weight similar to that of control mice (31). Surprisingly, ocular alterations such as cataracts and megaloglobus or buphthalmos appeared in old (more than 6 months) transgenic mice. RT-PCR analysis demonstrated the expression of the transgene (Figure $1 \mathrm{~A}$ ), indicating that tran-

A

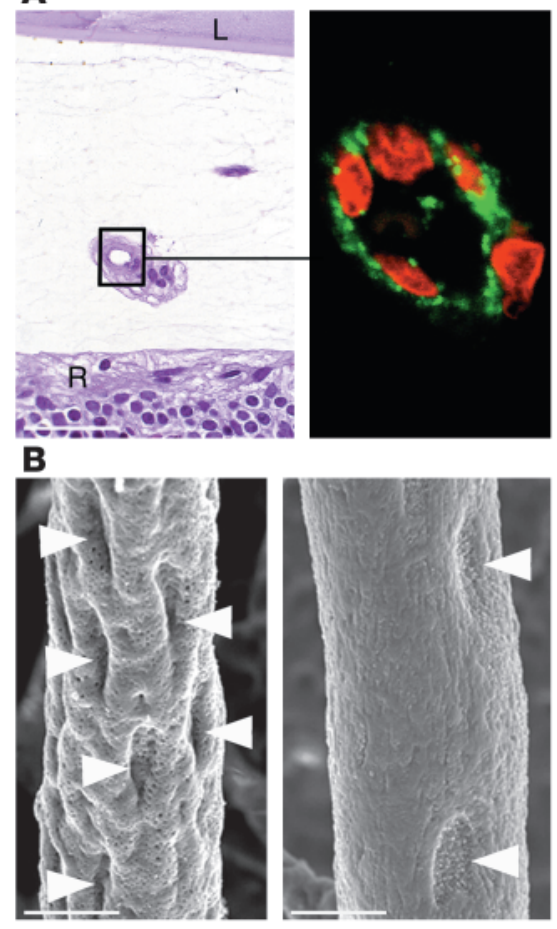


A

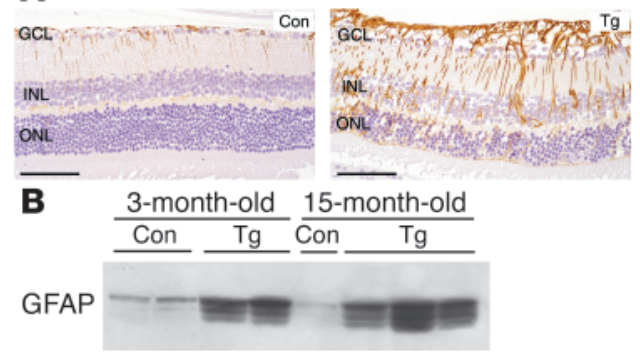

\section{Figure 7}

Structure of retina is altered in transgenic eyes. (A) Immunohistochemical analysis of GFAP expression in retina. Glial cells from transgenic retinas overexpressed GFAP. (B) Western blot analysis of GFAP protein in whole eye. Both 3- and 15-month-old transgenic mice showed high increase in GFAP protein. Scale bars: $81 \mu \mathrm{m}(\mathbf{A})$. scription from the RIP-I promoter was active in the eye of transgenic mice. This finding is consistent with the reported expression of preproinsulin in the eye (36). Northern blot analysis showed high IGF-1 expression in the retina (Figure 1B). In addition, by whole-mount in situ hybridization, IGF-1 expression was detected in the outer plexiform layer and the inner segment of photoreceptors of the transgenic retina (Figure 1, C and D). Thus, IGF-1 was overexpressed in photoreceptors of the transgenic eyes. This finding was parallel to high levels of IGF-1 in the aqueous humor of both 2- and 14-month-old transgenic eyes (Figure 2). Serum IGF-1 levels were similar in both control and transgenic mice (control, $300 \pm 20 \mathrm{ng} / \mathrm{ml}, n=8$, versus transgenic, $320 \pm 35$ $\mathrm{ng} / \mathrm{ml}, n=8)$, however. Furthermore, glucose concentration in the aqueous humor of both 2-and 12-month-old transgenic eyes was similar to that of controls (at 12 months: control, $126.1 \pm 18.1$ $\mathrm{mg} / \mathrm{dl}, n=5$, versus transgenic, $130.7 \pm 32.2 \mathrm{mg} / \mathrm{dl}, n=5)$ and was consistent with the normoglycemia of these mice (at 12 months: control, $138 \pm 8 \mathrm{mg} / \mathrm{dl}, n=7$, versus transgenic, $142 \pm 6 \mathrm{mg} / \mathrm{dl}, n=7$ ). This indicated that local ocular hyperglycemia was not responsible for the alterations in transgenic eyes.

Transgenic mice overexpressing IGF-1 develop nonproliferative retinopathy. Increased production of IGF-1 in the retina of transgenic mice led to the progressive development of all stages of diabetic-like retinopathy in both eyes. One-day-old transgenic mice did not present proliferation of hyaloid vessels in the eye (data not shown). In contrast, features of nonproliferative diabetic retinopathy were observed in 2 -month-old mice. Thus, transgenic eyes showed loss of pericytes (Figure 3). The quantitative study of retinal digest preparations showed a decrease of about $36 \%$ in the number of pericytes per square millimeter of capillary area (Figure 3, B and C). In addition, although we observed a slight decrease in the total endothelial cell number per square millimeter of capillary area in retinal digest preparations of transgenic eyes, the difference was not significant (Figure 3C). Nevertheless, after laser confocal microscopy analysis, several acellular capillaries were observed in the transgenic retina (Fig- ure 3A). Furthermore, thickening of the capillary basement membrane was also observed, as detected by increased collagen IV immunostaining (Figure 4A) and by transmission electron microscopy (Figure 4B). Collagen IV is also highly increased in human diabetic retinal blood vessels (37). Measurement of the capillary basal membrane thickness in transmission electron microscopy images of transgenic retinas showed an increase of about 1.8-fold (Figure 4C). Similarly, direct injection of IGF-1 into the vitreous cavity of pigs lead to significant capillary basement membrane thickening (38).

Transgenic mice overexpressing IGF-1 develop proliferative retinopathy. Transgenic mice aged 6 months and older presented altered retinal vascularization (Figure 5A) and showed most of the features found in human diabetic retinopathy, such as intraretinal microvascular abnormalities (IRMAs) and neovascularization (3). Venule dilatation (about 60\%) was noted in 6-month-old transgenic mice (control $30.6 \pm 0.8 \mu \mathrm{m}, n=8$, versus transgenic, $53.1 \pm 3.7$ $\mu \mathrm{m}, n=8 ; P<0.01)$. Furthermore, these mice showed IRMAs, such as intraretinal capillary vessels branching with an anomalous frequency and angulation (Figure 5B). Neovascularization was also observed in transgenic eyes since new vessels formed inside the retina (Figure 5, A, C, and D) and in the vitreous cavity (Figure 6). Preretinal new vessels appeared as looplike vessels that originated from veins and invaded areas of nonperfusion (Figure 5, A and C). Moreover, transgenic eyes showed dense areas of intraretinal neocapillaries (Figure 5D). Blood vessels were detected in the vitreous cavity of 6 - and 12-month-old transgenic mice, as confirmed by the presence of vWF, a marker of endothelial cells (Figure 6A). Injection of Mercox resin in the thoracic aorta demonstrated that intravitreous vessels were functional and connected to the general circulation. Consistent with the morphology of angiogenic vessels (39), casts of intravitreous vessels showed more prominent and numerous imprints of endothelial nuclei than intraretinal vessels (Figure 6B).

Transgenic retinas show increased GFAP expression in glial cells. Similarly to human diabetic retinopathy, in which GFAP is upregulated

\section{Figure 8}

The retina was folded and detached in the transgenic eye (left panel). Note the presence of vessels between retinal folds (inset magnified in upper right panel). Arrows point to endothelial cell nuclei. Stimulated macrophages, labeled with Griffonia simplicifolia lectin (green), were observed in the outer surface of detached flat-mounted transgenic retinas (lower right panel). Nuclei were counterstained with propidium iodide (red). The image is a single confocal section. $\mathrm{CH}$, choroid; PE, retinal pigment epithelium; S, sclera; SE, subretinal space formed by the separation between neuroretina and pigment epithelium. Scale bars: $354 \mu \mathrm{m}$ (left panel) and $16 \mu \mathrm{m}$ (right panel).

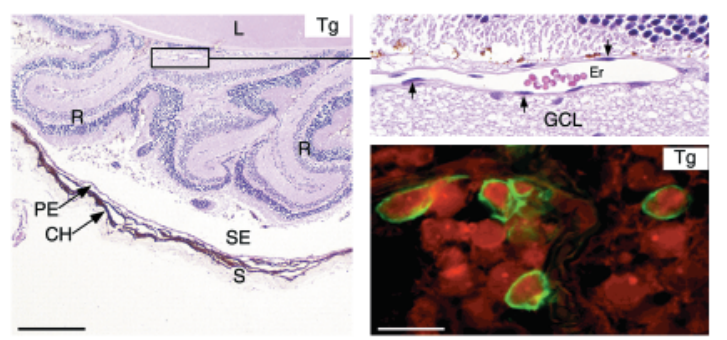


A

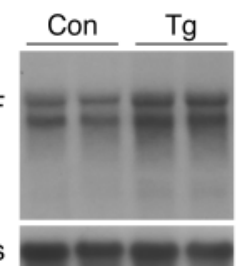

B

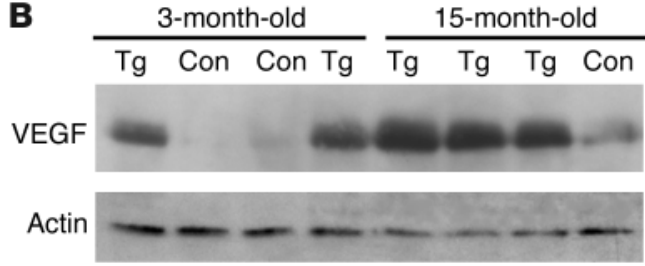

Figure 9

VEGF expression in the transgenic eye. (A) Total RNA was obtained from whole eyes and analyzed by Northern blot as indicated in Methods. Expression of VEGF transcripts was increased in 15-month-old transgenic mice. (B) Western blot analysis of VEGF protein in whole eye. Both 3- and 15-month-old transgenic mice showed a significant increase in VEGF protein. Actin documents equal loading.

in Müller cells (40), transgenic retinas overexpressed GFAP. This was detected by immunohistochemistry (Figure 7A) and Western blot analysis (Figure 7B). In control mice, GFAP staining was mainly confined to the ganglion cell layer around the preretinal vessels, corresponding to astrocytes and Müller cells. In contrast, transgenic mice showed highly increased GFAP in astrocytes and Müller cells, but Müller cells also expressed GFAP in the radial cytoplasmic processes extending throughout the retina (Figure $7 \mathrm{~A}$ ).

Old transgenic mice show retinal detachment. In advanced diabetic eye disease, neovascularization of the vitreous may form strong fibrous adhesions between it and the retina, which may contract and thus separate the neuroretina from the retinal pigment epithelium and lead to retinal detachment $(3,41)$. Transgenic retina overexpressing IGF-1 folded and detached in about $75 \%$ of eyes in mice more than 6 months old $(n=21)$ (Figure 8, left panel). Intravitreous vessels were observed between folds of transgenic detached retinas (Figure 8, inset). Macrophages in the subretinal space, a common feature in long-standing retinal detachment (42), were also observed in the transgenic eyes (Figure 8, right panel).

$I G F-1$ increases VEGF expression in the eye of transgenic mice. VEGF is a potent angiogenic factor, and IGF-1 may induce its expression in vitro (29). To examine whether the vascular alterations observed in transgenic eyes also correlated with an increase in VEGF, we measured VEGF by Northern blot and Western blot analysis. About a twofold increase in VEGF transcripts in transgenic eyes was detected (Figure 9A). Furthermore, a high increase in VEGF protein was also found in both 3- and 15-month-old transgenic eye extracts compared with controls (Figure 9B). These findings correlated with increased numbers of VEGF-expressing retinal cells after immunohistochemical analysis of flat-mounted retinas (Figure 10A). In humans proliferative diabetic retinopathy glial cells produce VEGF (43). Similarly, glial cells were in the transgenic retina cells overexpressing VEGF because they also coexpressed GFAP (Figure 10B).

Transgenic mice develop rubeosis iridis and neovascular glaucoma. The increase of IGF-1 in the eyes of transgenic mice was parallel to neo-

\section{Figure 10}

Immunohistochemical analysis of VEGF. (A) Only the flat-mounted 6-month-old transgenic retinas showed extensive groups of cells producing VEGF (red). Inset shows the location in the retina. (B) VEGF (red) was detected in GFAP-expressing glial cells (green). Scale bars: $44 \mu \mathrm{m}(\mathbf{A})$ and $23 \mu \mathrm{m}(\mathbf{B})$. vascularization of the iris (rubeosis iridis) (Figure 11). New vessels from the anterior face of the iris invaded corneal stroma, producing anterior synechias, the adhesions between the iris and the cornea (Figure 11A). Anterior synechias hampered drainage of aqueous humor in the iridocorneal angle and led to buphthalmos (Figure 11B, left panel). Eyes from 6-monthold mice showed a high increase in the size of the anterior chamber of the eyeball (Figure 11B, right panels) and in aqueous humor weight (about 2.6-fold) (control $2.0 \pm 0.3 \mathrm{mg}, n=8$, versus transgenic, $5.2 \pm 0.5$ $\mathrm{mg}, n=14 ; P<0.01)$. Transgenic mice also showed an increase in eye weight (control $22.7 \pm 0.4 \mathrm{mg}, n=8$, versus transgenic, $34.0 \pm 0.8 \mathrm{mg}, n=14 ; P<0.0001)$. Nevertheless, the aqueous humor/eye-weight ratio was higher (about $74 \%$ ) in transgenic mice. When IOP was determined in 6-month-old mice, a decrease (about $37 \%$ ) was observed in transgenic eyes (control, $6 \pm 0.6 \mathrm{mmHg}, n=8$, versus transgenic, $3.8 \pm 0.7 \mathrm{mmHg}(n=5) ; P<0.05)$. This agreed with the fact that a period of prolonged rise of IOP may be followed by a return to normotension or even hypotension (44). All these findings suggested that transgenic mice probably developed glaucoma. In addition, scanning electron microscopic analysis of critical-point dried transgenic eyes showed globular cells blocking the iridocorneal angle, while control eyes presented a trabecular meshwork free of these cells (Figure 11C). This may have reduced the outflow of aqueous humor. Furthermore, separation of ganglion cell nuclei greater than $20-30 \mu \mathrm{m}$ indicates ganglion cell loss, a feature of glaucoma $(45,46)$. In contrast to control retinas, most transgenic retinas of 12-month-old mice presented highly separated (greater than $30 \mu \mathrm{m}$ ) ganglion cell nuclei (Figure 12), which suggested that these mice may have developed glaucoma.

Transgenic mice develop cataracts. Cataracts, opacities in any part of the lens, are frequent in diabetic patients $(1,47)$ and were observed in about $85 \%(n=16)$ of 6 -month-old transgenic mice after lens extraction (Figure 13A, left panel). Moreover, at 12 months all transgenic mice showed obvious cataracts by external observation (Figure 13A, right panel). Histological analysis of lenses with cataracts showed that the opacity resulted from proliferation and migration of epithelial cells to the subcortical area (Figure 13B).

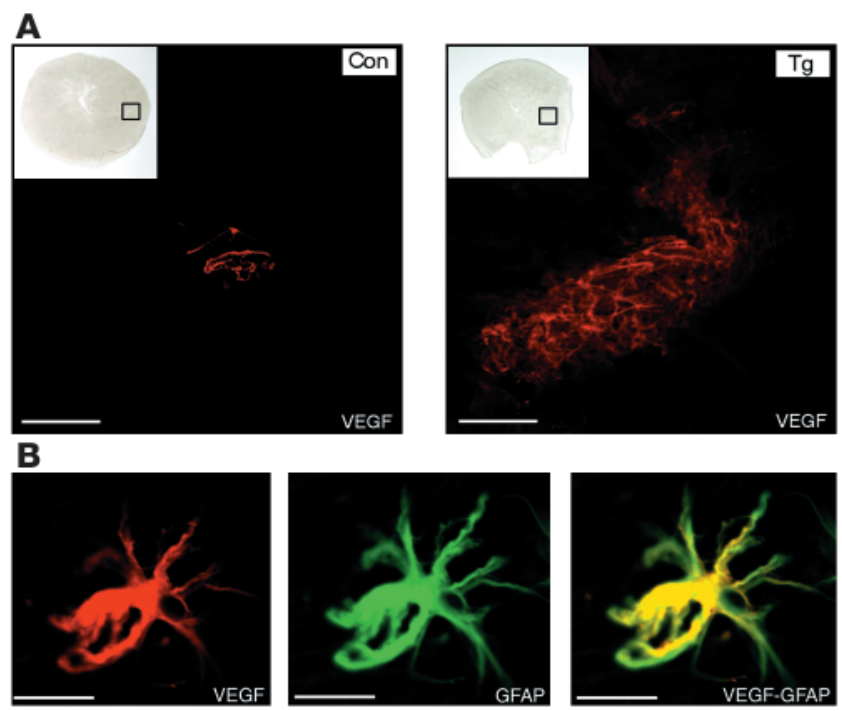


A

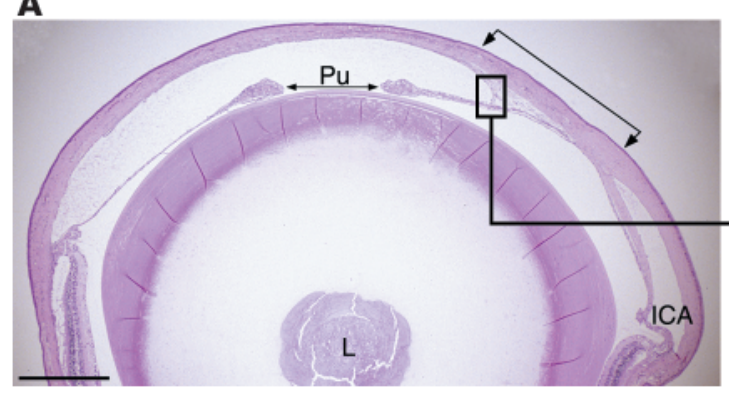

B

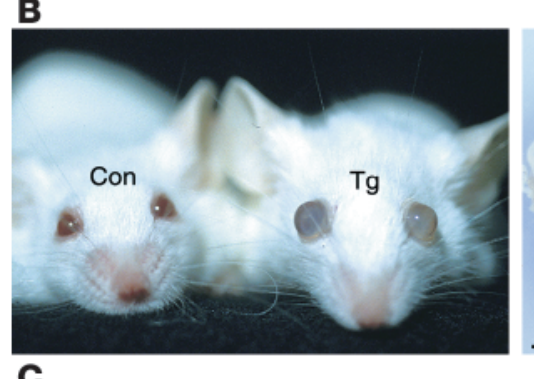

c
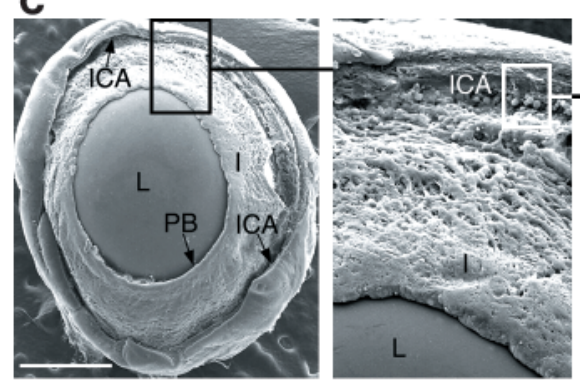
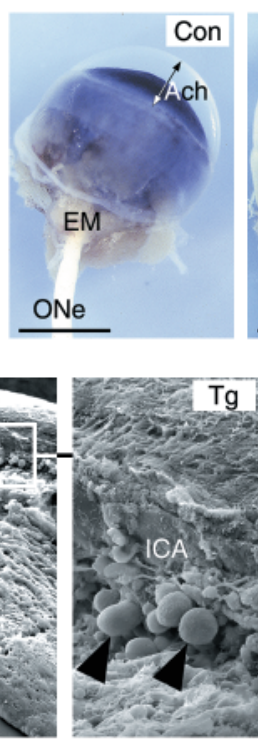
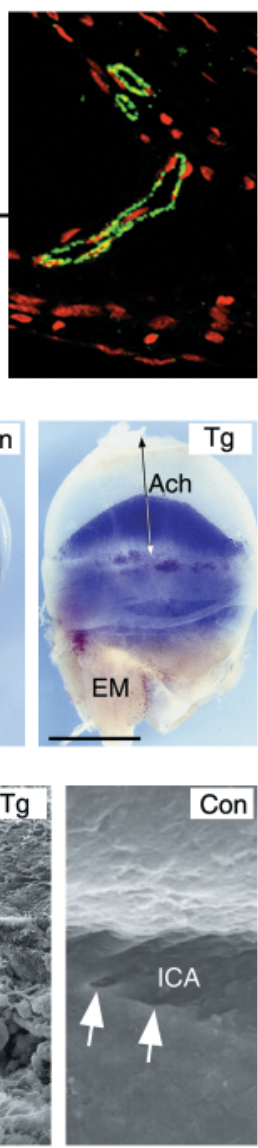

\section{Figure 11}

Transgenic mice develop rubeosis iridis and neovascular glaucoma. (A) The transgenic eye developed synechias (two connected arrows) that hampered the drainage of aqueous humor (left panel). Neovessels from the iris, marked with anti-vWF (green), invade the cornea (right panel, magnification of inset in left panel). (B) Transgenic mice developed buphthalmos. The length of transgenic anterior chamber was higher than that in controls. (C) Scanning electron microscopy analysis of iridocorneal angle. (Insets in left two panels are magnified in the panels to their right.) Transgenic eyes showed an accumulation of cells (arrowheads) occluding the trabecular meshwork. In contrast, control mice presented an unobstructed trabecular meshwork (arrows). Ach, anterior chamber; EM, extraocular muscles; I, iris; ICA, iridocorneal angle; ONe, optic nerve; PB, pupillary border; Pu, pupil opening. Scale bars: $460 \mu \mathrm{m}$ (A), 2,100 $\mu \mathrm{m}$ (B), and $900 \mu \mathrm{m}$ (C).

\section{Discussion}

This study indicates a direct relationship of IGF-1 with the development of ocular alterations resembling those found in human diabetic eye disease. Since transgenic mice were normoglycemic and normoinsulinemic and had normal levels of circulating IGF-1, these alterations were probably triggered by IGF-1 overexpression in the retina. Thus, in these transgenic mice, local production of IGF-1 rather than hyperglycemia or hyperinsulinemia led to ocular alterations. These mice showed local expression of IGF-1 in the neuronal cells of the eye from the RIP, which was consistent with the expression of preproinsulin in adult retinas (36). Furthermore, it has recently been shown that insulin receptors are present in endothelial cells of the retina and that insulin signaling may play a role in retinal neovascularization (10). Thus, in the IGF-1 transgenic eye, the concurrence of (a) direct action of IGF-1 through binding to IGF-1 receptor, and to a lesser extent to the insulin receptor, and (b) indirect action through induction of other growth factors such as VEGF, led to a cascade of events that resulted in retinopathy, rubeosis iridis, neovascular glaucoma, and cataracts as the animals aged.

\section{Figure 12}

Ganglion cell loss in transgenic retina. Histological sections of retina from 12-month-old transgenic mice showed decreased number of retinal ganglion cells (arrows). Scale bars: $45 \mu \mathrm{m}$.
The role of IGF-1 in nonproliferative stages of retinopathy is not clear. In the retina of eye donors with short diabetes duration the expression of IGF-1 is reduced (48). In addition, in the retinas of rats with 2-month duration of STZ-induced diabetes, IGF-1 mRNA levels are similar to those of controls, but after 5 months of diabetes they fail to increase to the levels recorded in age-matched controls (48). Similarly, no changes from control values were reported in $\mathrm{BB} / \mathrm{W}$ rats who have had diabetes for 3 months (49). A decrease in rat retinal IGF-1 mRNA levels 3 weeks after STZ-induced diabetes has also been described, however (50). In contrast, IGF-1 receptor levels were increased in human or unchanged in rat diabetic retinas (48). We found that overexpression of IGF-1 in the normoglycemic transgenic eyes led to alterations similar to those found in early stages of human diabetic retinopathy, such as pericyte loss and thickening of capillary basement membrane. It has been shown in pancreatic $\beta$ cells (INS-1) or in human renal fibroblasts that there is cooperation between glucose and IGF-1. Thus, low levels of IGF-1 in the presence of increasing glucose concentrations exerts effects on $\beta$ cell mitogenesis similar to low glucose concentration in the presence of increas- 
A
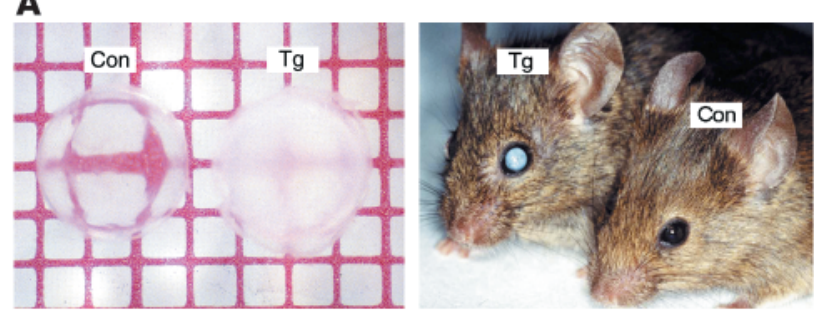

B
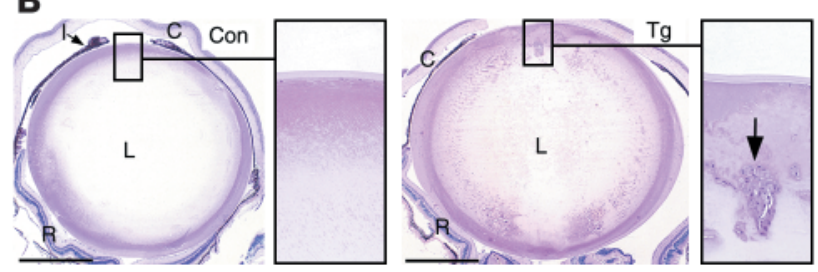

ing IGF-1 levels (51). In human renal fibroblasts, IGF-1 also has a synergistic effect with glucose on the induction of collagens (52). Thus, there may also be cooperative action between glucose and IGF-1 in the development of nonproliferative stages of retinopathy. In our nondiabetic transgenic model, the increase in ocular IGF-1 under normoglycemia might lead to ocular alterations similar to those of hyperglycemia in the presence of low ocular IGF-1.

In 2- to 3-month-old IGF-1 transgenic mice, VEGF expression increased in glial cells in the retina, and this was maintained in older mice. This increase was consistent with IGF-1 induction of VEGF transcription (29). Furthermore, the presence of areas of nonvascular perfusion in the transgenic retina of IGF-1 mice may have also increased VEGF, as reported (53). Thus, neovascularization may have arisen as a result of the concerted action of IGF-1 and VEGF. It has been suggested that IGF-1 is essential in retinal neovascularization, and interactions between IGF-1 and IGF-1 receptor are necessary for induction of maximal neovascularization by VEGF, since treatment with an IGF-1 receptor antagonist suppresses retinal neovascularization in vivo (9). Similarly, after hypoxia, VENIFARKO mice show reduced neovascularization of retina despite increased VEGF (10). This suggests that the lack of IGF-1 signaling prevents neovascularization and is consistent with a cooperative action of VEGF and IGF-1 in this process (10). Furthermore, VEGF does not stimulate normal retinal vascular development in the absence of IGF-1 (28). Taken together, these findings suggest that IGF-1 may modulate VEGF action in retinal neovascularization. The neovascularization in the IGF-1 transgenic eyes was mainly located in the retina and inside the vitreous cavity, producing retinal detachment, as occurs in human proliferative diabetic retinopathy (41). Although transgenic mice overexpressing VEGF $(26,27)$ in photoreceptors under the control of the rhodopsin promoter also show neovascularization and detachment of the retina, the new vessels invade the subretinal space (26), which is not found in our transgenic mice. This may result from VEGF expression in photoreceptors, while in IGF-1-expressing mice VEGF was produced in glial cells, as in diabetic retinopathy.

The IGF-1 produced in the transgenic retina accumulated in the aqueous humor and was thus available to IGF-1 receptors in the iris (8), probably leading to rubeosis iridis. VEGF production from the transgenic retina may also have contributed to this process. VEGF is considered a key factor in the development of rubeosis iridis in nonhuman primates (54). Iris neovessels produced anterior synechias in the transgenic eyes, which may have been responsi-

\section{Figure 13}

Transgenic mice develop cataracts. (A) Lens from transgenic mice developed opacities (left panel), which were seen macroscopically in all 12-month-old mice (right panel). (B) Histopathological analysis of transgenic lens showed migration of epithelial cells toward the lens subcortical area (arrow). (Insets are magnified in the corresponding rectangles.) C, cornea. Scale bar: $670 \mu \mathrm{m}$.

ble for the glaucoma and consequent ganglion cell loss. These IGF-1-expressing mice are the first transgenic model to develop iris neovascularization and neovascular glaucoma. Laser occlusion of retinal veins is a reliable way to produce iris neovascularization, but the neovessels regress 21-28 days later (55).

Lens epithelial cells have IGF-1 receptors, and IGF-1 binding stimulates lens cell differentiation and proliferation $(56,57)$. Thus, the IGF-1 in the aqueous humor of transgenic eyes may have induced proliferation and migration of epithelial lens cells to the subcortical area, producing lens opacity. Cataracts are also a consequence of the aggregation of lens proteins (58). The lens fibers contain $\alpha-, \beta$-, and $\gamma$-crystallin, the first of which protects against protein aggregation (58). It has been described that IGF-1 increases the overall ratio of $\beta$ - and $\gamma$-crystallin to $\alpha$-crystallin in the fiber cell, which could predispose the lens to cataracts (59). IGF-1 may have altered crystallins in transgenic eyes and thus also contributed to cataract formation.

In summary, transgenic mice expressing IGF-1 in the retina mimic most features of human diabetic eye disease. This suggests that IGF-1 may have a role in the development of the ocular complications. Although the source of ocular IGF-1 in diabetic retinopathy is unknown, the increase in this growth factor, either from serum or endogenously produced, probably cooperates with glucose, VEGF, and/or other growth factors and leads to progression of diabetic eye disease. In addition, IGF-1 transgenic animals provide a rodent model of diabetic eye disease in the absence of systemic metabolic defects due to the lack of insulin or to insulin resistance. Thus, these transgenic mice may provide a valid model in which to examine the molecular mechanisms that lead to the ocular alterations of diabetes. They may also be very useful in designing and assaying new therapies for diabetic eye disease, including nonproliferative and proliferative retinopathy, rubeosis iridis, neovascular glaucoma, and cataracts.

\section{Acknowledgments}

We thank C.H. Ros, M. Moya, and V. Melgarejo for technical assistance. E. Ayuso was recipient of a predoctoral fellowship from Direcció General de Recerca, Generalitat de Catalunya. A. Bosch was recipient of a "Ramon y Cajal" contract, from Spain's Ministry of Science and Technology. This work was supported by grants from European Foundation for the Study of Diabetes/Juvenile Diabetes Research Foundation/Novo Nordisk, Instituto de Salud Carlos III (FIS98/1063, G03/212, and C03/08, awarded to F. Bosch), and from La Marató de TV3 Foundation.

Received for publication July 14, 2003, and accepted in revised form February 10, 2004.

Address correspondence to: Fatima Bosch, Center of Animal Biotechnology and Gene Therapy, Universitat Autònoma de Barcelona, E-08193, Bellaterra, Spain. Phone: 34-93-5814182; Fax: 34-93-5814180; E-mail: fatima.bosch@uab.es.

Jesús Ruberte and Eduard Ayuso contributed equally to this work. 
1. Forrester, J.V., and Knott, R.M. 1997. Pathogenesis of diabetic retinopathy. In Textbook of diabetes. J. Pickup and G. Williams, editors. Blackwell Science Ltd. Oxford, United Kingdom. 45.1-45.19.

2. Porta, M., and Bandello, F. 2002. Diabetic retinopathy: a clinical update. Diabetologia. 45:1617-1634.

3. Aiello, L.P., et al. 1998. Diabetic retinopathy. Diabetes Care. 21:143-156.

4. Smith, L.E., et al. 1994. Oxygen-induced retinopathy in the mouse. Invest. Ophthalmol. Vis. Sci. 35:101-111.

5. Merimee, T.J., Zapf, J., and Froesch, E.R. 1983. Insulin-like growth factors. Studies in diabetics with and without retinopathy. N. Engl. J. Med. 309:527-530.

6. Grant, M., Russell, B., Fitzgerald, C., and Merimee, T.J. 1986. Insulin-like growth factors in vitreous. Studies in control and diabetic subjects with neovascularization. Diabetes. 35:416-420.

7. Inokuchi, N., et al. 2001. Vitreous levels of insulinlike growth factor-I in patients with proliferative diabetic retinopathy. Curr. Eye Res. 23:368-371.

8. Lambooij, A.C., et al. 2003. Insulin-like growth factor-I and its receptor in neovascular age-related macular degeneration. Invest. Ophthalmol. Vis. Sci. 44:2192-2198.

9. Smith, L.E., et al. 1999. Regulation of vascular endothelial growth factor-dependent retinal neovascularization by insulin-like growth factor-1 receptor. Nat. Med. 5:1390-1395.

10. Kondo, T., et al. 2003. Knockout of insulin and IGF-1 receptors on vascular endothelial cells protects against retinal neovascularization. J. Clin. Invest. 111:1835-1842. doi:10.1172/JCI200317455.

11. Hellstrom, A., et al. 2002. IGF-I is critical for normal vascularization of the human retina. J. Clin. Endocrinol. Metab. 87:3413-3416.

12. Burren, C.P., Berka, J.L., Edmondson, S.R., Werther, G.A., and Batch, J.A. 1996. Localization of mRNAs for insulin-like growth factor-I (IGF-I), IGF-I receptor, and IGF binding proteins in rat eye. Invest. Ophthalmol. Vis. Sci. 37:1459-1468.

13. Lauszus, F.F., Klebe, J.G., Bek, T., and Flyvbjerg, A. 2003. Increased serum IGF-I during pregnancy is associated with progression of diabetic retinopathy. Diabetes. 52:852-856.

14. Dahl-Jorgensen, K., Brinchmann-Hansen, O., Hanssen, K.F., Sandvik, L., and Aagenaes, O. 1985. Rapid tightening of blood glucose control leads to transient deterioration of retinopathy in insulin dependent diabetes mellitus: the Oslo study. $\mathrm{Br}$. Med. J. (Clin. Res. Ed.) 290:811-815.

15. Hanssen, K.F., Dahl-Jorgensen, K., and Brinchmann-Hansen, O. 1985. The influence of strict control on diabetic complications. Acta Endocrinol. Suppl. (Copenh.) 272:57-60.

16. The Diabetes Control and Complications Trial Research Group. 1993. The effect of intensive treatment of diabetes on the development and progression of long-term complications in insulin-dependent diabetes mellitus. N. Engl. J. Med. 329:977-986.

17. Moskalets, E., Galstyan, G., Starostina, E., Antsiferov, M., and Chantelau, E. 1994. Association of blindness to intensification of glycemic control in insulin-dependent diabetes mellitus. J. Diabetes Complications. 8:45-50.

18. Diabetes Control and Complications Trial Research Group. 1995. Progression of retinopathy with intensive versus conventional treatment in the Diabetes Control and Complications Trial. Ophthalmology. 102:647-661.

19. 1995. The effect of intensive diabetes treatment on the progression of diabetic retinopathy in insulindependent diabetes mellitus. The Diabetes Control and Complications Trial. Arch. Ophthalmol. 113:36-51.

20. Chantelau, E., and Kohner, E.M. 1997. Why some cases of retinopathy worsen when diabetic control improves. BMJ. 315:1105-1106.

21. Smith, L.E., et al. 1997. Essential role of growth hormone in ischemia-induced retinal neovascularization. Science. 276:1706-1709.

22. Aiello, L.P., et al. 1994. Vascular endothelial growth factor in ocular fluid of patients with diabetic retinopathy and other retinal disorders. N. Engl. J. Med. 331:1480-1487.

23. Adamis, A.P., et al. 1994. Increased vascular endothelial growth factor levels in the vitreous of eyes with proliferative diabetic retinopathy. Am.J. Ophthalmol. 118:445-450.

24. Malecaze, F., et al. 1994. Detection of vascular endothelial growth factor messenger RNA and vascular endothelial growth factor-like activity in proliferative diabetic retinopathy. Arch. Ophthalmol. 112:1476-1482.

25. Boulton, M., Foreman, D., Williams, G., and McLeod, D. 1998. VEGF localisation in diabetic retinopathy. Br. J. Ophthalmol. 82:561-568.

26. Okamoto, N., et al. 1997. Transgenic mice with increased expression of vascular endothelial growth factor in the retina: a new model of intraretinal and subretinal neovascularization. Am. J. Pathol. 151:281-291.

27. Ohno-Matsui, K., et al. 2002. Inducible expression of vascular endothelial growth factor in adult mice causes severe proliferative retinopathy and retinal detachment. Am. J. Pathol. 160:711-719.

28. Hellstrom, A., et al. 2001. Low IGF-I suppresses VEGF-survival signaling in retinal endothelial cells: direct correlation with clinical retinopathy of prematurity. Proc. Natl. Acad. Sci. U. S. A. 98:5804-5808.

29. Punglia, R.S., et al. 1997. Regulation of vascular endothelial growth factor expression by insulin-like growth factor I. Diabetes. 46:1619-1626.

30. Miele, C., Rochford, J.J., Filippa, N., Giorgetti-Peraldi, S., and Van Obberghen, E. 2000. Insulin and insulin-like growth factor-I induce vascular endothelial growth factor mRNA expression via different signaling pathways. J. Biol. Chem. 275:21695-21702.

31. George, M., et al. 2002. $\beta$ cell expression of IGF-I leads to recovery from type 1 diabetes. J. Clin. Invest. 109:1153-1163. doi:10.1172/JCI200212969.

32. Nieto, M.A., Patel, K., and Wilkinson, D.G. 1996. In situ hybridization analysis of chick embryos in whole mount and tissue sections. Methods Cell Biol. 51:219-235.

33. Hammes, H.P., Martin, S., Federlin, K., Geisen, K., and Brownlee, M. 1991. Aminoguanidine treatment inhibits the development of experimental diabetic retinopathy. Proc. Natl. Acad. Sci. U. S. A. 88:11555-11558.

34. Danias, J., Kontiola, A.I., Filippopoulos, T., and Mittag, T. 2003. Method for the noninvasive measurement of intraocular pressure in mice. Invest. Ophthalmol. Vis. Sci. 44:1138-1141.

35. Cuthbertson, R.A., and Mandel, T.E. 1986. Anatomy of the mouse retina. Capillary basement membrane thickness. Invest. Ophthalmol. Vis. Sci. 27:1653-1658.

36. Budd, G.C., Pansky, B., and Glatzer, L. 1993. Preproinsulin mRNA in the rat eye. Invest. Ophthalmol. Vis. Sci. 34:463-469.

37. Roy, S., Maiello, M., and Lorenzi, M. 1994. Increased expression of basement membrane collagen in human diabetic retinopathy. J. Clin. Invest. 93:438-442.

38. Danis, R.P., and Bingaman, D.P. 1997. Insulin-like growth factor-1 retinal microangiopathy in the pig eye. Ophthalmology. 104:1661-1669.

39. Peao, M.N., Aguas, A.P., de Sa, C.M., and Grande, N.R. 1994. Neoformation of blood vessels in association with rat lung fibrosis induced by bleomycin. Anat. Rec. 238:57-67.

40. Mizutani, M., Gerhardinger, C., and Lorenzi, M.
1998. Muller cell changes in human diabetic retinopathy. Diabetes. 47:445-449.

41. Dowler, J.G.F., and Hamilton, A.M.P. 1997. Clinical features of diabetic eye disease. In Textbook of diabetes. J. Pickup and G. Williams, editors. Blackwell Science Ltd. Oxford, United Kingdom. 46.1-46.15.

42. Smith, R.S., Hawes, N.L., Chang, B., and Nishina, P.M. 2002. Retina. In Systematic evaluation of the mouse eye. R.S. Smith, editor. CRC Press. Boca Raton, Florida, USA. 195-225.

43. Amin, R.H., et al. 1997. Vascular endothelial growth factor is present in glial cells of the retina and optic nerve of human subjects with nonproliferative diabetic retinopathy. Invest. Ophthalmol. Vis. Sci. 38:36-47.

44. Ritch, R., Shields, M.B., and Krupin, T. 1996. Ocular hypotony. In The glaucomas. 2 nd edition. Mosby Publishing Co. St. Louis Missouri, USA.

45. Smith, R.S., John, S.W.M., and Nishina, P.M. 2002. Posterior segment and orbit. In Systematic evaluation of the mouse eye. R.S. Smith, editor. CRC Press. Boca Raton, Florida, USA. 25-44.

46. Quigley, H.A., Addicks, E.M., Green, W.R., and Maumenee, A.E. 1981. Optic nerve damage in human glaucoma. II. The site of injury and susceptibility to damage. Arch. Ophthalmol. 99:635-649.

47. Klein, B.E., Klein, R., and Moss, S.E. 1985. Prevalence of cataracts in a population-based study of persons with diabetes mellitus. Ophthalmology. 92:1191-1196.

48. Gerhardinger, C., McClure, K.D., Romeo, G., Podesta, F., and Lorenzi, M. 2001. IGF-I mRNA and signaling in the diabetic retina. Diabetes. 50:175-183.

49. Charkrabarti, S., Ghahary, A., Murphy, L.J., and Sima, A.A. 1991. Insulin-like growth factor-I expression is not increased in the retina of diabetic BB/Wrats. Diabetes Res. Clin. Pract. 14:91-97.

50. Lowe, W.L., Jr., Florkiewicz, R.Z., Yorek, M.A., Spanheimer, R.G., and Albrecht, B.N. 1995. Regulation of growth factor mRNA levels in the eyes of diabetic rats. Metabolism. 44:1038-1045.

51. Hugl, S.R., White, M.F., and Rhodes, C.J. 1998. Insulin-like growth factor I (IGF-I)-stimulated pancreatic beta-cell growth is glucose-dependent. Synergistic activation of insulin receptor substrate-mediated signal transduction pathways by glucose and IGF-I in INS-1 cells. J. Biol. Chem. 273:17771-17779.

52. Lam, S., et al. 2003. Connective tissue growth factor and IGF-I are produced by human renal fibroblasts and cooperate in the induction of collagen production by high glucose. Diabetes. 52:2975-2983.

53. Pierce, E.A., Avery, R.L., Foley, E.D., Aiello, L.P., and Smith, L.E. 1995. Vascular endothelial growth factor/vascular permeability factor expression in a mouse model of retinal neovascularization. Proc. Natl. Acad. Sci. U. S. A. 92:905-909.

54. Tolentino, M.J., et al. 1996. Vascular endothelial growth factor is sufficient to produce iris neovascularization and neovascular glaucoma in a nonhuman primate. Arch. Ophthalmol. 114:964-970.

55. Miller, J.W. 1997. Vascular endothelial growth factor and ocular neovascularization. Am. J. Pathol. 151:13-23.

56. Ibaraki, N., Lin, L.R., and Reddy, V.N. 1996. A study of growth factor receptors in human lens epithelial cells and their relationship to fiber differentiation. Exp. Eye Res. 63:683-692.

57. Klok, E., Lubsen, N.H., Chamberlain, C.G., and McAvoy, J.W. 1998. Induction and maintenance of differentiation of rat lens epithelium by FGF-2, insulin and IGF-1. Exp. Eye Res. 67:425-431.

58. Horwitz, J. 2003. Alpha-crystallin. Exp. Eye Res. 76:145-153.

59. Civil, A., van Genesen, S.T., Klok, E.J., and Lubsen, N.H. 2000. Insulin and IGF-I affect the protein composition of the lens fibre cell with possible consequences for cataract. Exp. Eye Res.70:785-794. 